\title{
Avaliação dos Fatores Oficiais de Correção da Produção até o Primeiro Controle Leiteiro em Vacas Holandesas ${ }^{1}$
}

\section{Nilson Nunes Morais Júnior ${ }^{2}$, Rilke Tadeu Fonseca de Freitas ${ }^{3}$, Marcos Neves Pereira ${ }^{3}$, Antonio Ilson Gomes de Oliveira ${ }^{4}$, Idalmo Garcia Pereira ${ }^{5}$, Júlio Sílvio de Souza Bueno Filho ${ }^{3}$, Tarcísio de Moraes Gonçalves ${ }^{6}$}

RESUMO - Para o cálculo oficial do desempenho de vacas Holandesas no Brasil, com base em controles leiteiros mensais, são utilizados os fatores de correção propostos por Everett \& Carter (1968) para estimar a produção parcial do parto ao primeiro controle diário de uma dada lactação. A adequacidade destes fatores foi avaliada em 262 lactações oriundas de dois rebanhos que adotavam controle diário da produção de leite por vaca. Os dados foram divididos em classes com base na idade ao parto (CIP): CIP 1 (até 3 anos), CIP 2 (3,1 a 4 anos), CIP 3 (4,1 a 5 anos), CIP 4 (5,1 a 6 anos) e CIP 5 (acima de 6,1 anos) com 104, 56, 44, 18 e 40 lactações, respectivamente. A produção de leite do parto até um dia pré-definido da lactação foi estimada com os fatores oficiais e assumindo que o primeiro controle da produção naquela lactação ocorreu nos dias $6^{\circ} \stackrel{\circ}{-}, 20^{\circ}$, $, 34^{\circ} \stackrel{\circ}{ }, 48^{\circ}$ o, $62^{\circ}$ ou $75^{\circ}$. Os dados estimados foram comparados aos dados reais calculados com a pesagem diária do leite. Em todas as CIP, os fatores oficiais superestimaram a produção acumulada até o primeiro controle e o erro relativo de superestimação decresceu com o avançar do dia do primeiro controle. A quantidade superestimada de leite no período aumentou com o avançar do dia do primeiro controle e foi 13,9 e 87,1 kg na CIP 1 e 20,6 e 196,3 kg na CIP 2 para os dias 6o e 75으, respectivamente. As CIPs 3, 4 e 5 apresentaram valores intermediários. Novos fatores para dias do primeiro controle variando de 6 a 75 e nas 5 CIPs foram gerados pela mesma metodologia descrita por Everett \& Carter (1968). Os fatores gerados foram menores que os importados e rotineiramente utilizados para cálculo oficial de lactações no Brasil.

Palavras-chave: vacas Holandesas, Brasil, produção de leite

\section{Evaluation of Correction Factors Used to Estimate Milk Production from Calving to the First Monthly Control}

\begin{abstract}
Official Holstein cow performance data in Brazil, based on monthly milk weights, is calculated using the correction factors proposed by Everett \& Carter (1968) to estimate the partial production from calving to the first daily control in a given lactation. The adequacy of these factors was evaluated using 262 lactations from two herds with daily control of milk production per cow. Data were divided into classes based on age at calving (CIP): CIP 1 (up to 3 years), CIP 2 ( 3.1 to 4 years), CIP 3 ( 4.1 to 5 years), CIP 4 (5.1 to 6 years) and CIP 5 (above 6.1 years) which included 104, 56, 44, 18 and 40 lactations, respectively. Milk yield from calving to a set day of lactation was estimated with the official factors and assuming that the first control of production of that given lactation occurred on days $6^{\text {th }}, 20^{\text {th }}, 34^{\text {th }}, 48^{\text {th }}, 62^{\text {nd }}$ or $75^{\text {th }}$. Estimated data was compared with real data calculated from daily milk weights. For all CIPs, official factors overestimated the production from calving to first control and the relative overestimation error decreased with advance in the first control day. The overestimated amount of milk in that period increased with advance in the first control day and was 13.9 and 87.1 $\mathrm{kg}$ in CIP 1 and 20.6 and $196.3 \mathrm{~kg}$ in CIP 2 for days $6^{\text {th }}$ and $75^{\text {th }}$, respectively. CIPs 3,4 , and 5 had intermediate values. New factors for first control days ranging from 6 to 75 and in all 5 CIPs were generated using the same methodology described by Everett \& Carter (1968). The generated factors were smaller than the ones imported and routinely used to calculate official lactation records in Brazil.
\end{abstract}

Key Words: Holstein cows, Brazil, milk production

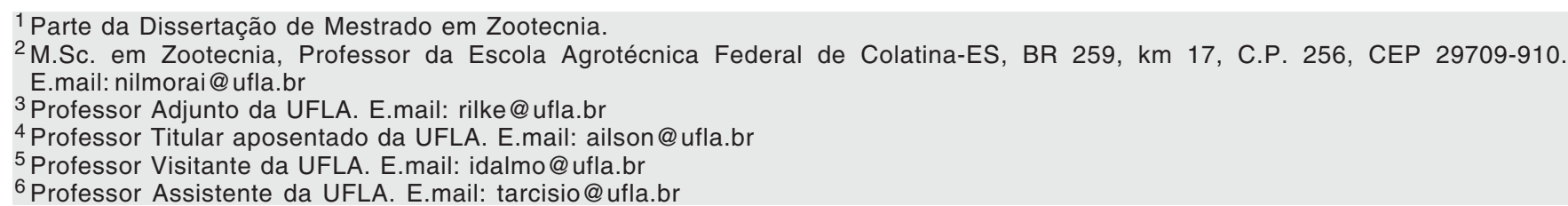




\section{Introdução}

O controle da produção de leite em animais da raça Holandesa vem sendo supervisionado desde 1946 pela Associação Brasileira de Criadores - ABC e, mais recentemente, pela Associação Brasileira de Criadores de Bovinos da Raça Holandesa - ABCBRH, ambas oficialmente reconhecidas pelo Ministério da Agricultura (Machado, 1997). Esse controle tem como finalidades nacionais e institucionais: aquilatar o potencial produtivo, coletar dados estatísticos para teste de progênie e melhoramento genético e divulgar os resultados atualizados para a comunidade.

No Brasil, o controle leiteiro oficial é realizado mensalmente e as pesagens são efetuadas em intervalos regulares, entre controles consecutivos, sendo permitidos intervalos de 15 a 45 dias. O primeiro controle da lactação é realizado oficialmente entre o 60 e 75으 dia após o parto.

O total de leite produzido em cada lactação é estimado por uma equação matemática contendo fatores propostos por Everett \& Carter (1968). Como na maioria das vezes não é possível iniciar o controle logo após o parto, nessa equação são utilizados fatores de correção que permitem estimar a produção de leite da vaca do parto até o dia do 1 o controle. Dessa forma, a produção de leite no primeiro controle é multiplicada pelo número de dias que o separa do parto e pelo fator de ajuste para o mesmo. Esses fatores de correção foram estimados por Everett \& Carter (1968), baseandose em controles diários de 403 lactações completas, realizadas entre junho de 1964 e outubro de 1967, no rebanho de gado Holandês da Universidade de Cornell -EUA.

Considerando-se as condições climáticas brasileiras e as características zootécnicas próprias de nossos rebanhos no que se refere aos sistemas de produção, alimentação e manejo, níveis de produção e composição genética, é possível que o emprego desses fatores de ajuste em controles leiteiros no Brasil possam estar causando erros no cálculo da produção total na lactação das vacas controladas.

Objetivou-se, com este estudo, avaliar os efeitos do emprego direto dos fatores de correção oficiais na estimação da produção de leite do parto até o dia do primeiro controle, comparada a produções reais obtidas de dados diários de produção de vacas da raça Holandesa sob controle leiteiro no Brasil.

R. Bras. Zootec., v.31, n.2, p.924-933, 2002 (suplemento)

\section{Material e Métodos}

Os dados utilizados neste estudo são originários de controles diários de produção provenientes de dois rebanhos da raça Holandesa, que participam do controle leiteiro oficial junto à Associação de Criadores de Gado Holandês de Minas Gerais.

O rebanho denominado "Cova da Onça", localizado no município de Pedro do Rio - RJ, participou neste estudo com 163 lactações oriundas do período de 04/ 94 a 12/99. Do segundo rebanho, denominado "EPAMIG", localizado no município de Três Pontas MG, foram utilizadas 99 lactações correspondentes ao período de 04/97 a 12/2000.

O estudo foi conduzido no Departamento de Zootecnia da Universidade Federal de Lavras. A fase inicial constou da verificação dos dados diários de produção, eliminando-se lactações com falhas ou alterações drásticas de produção nos primeiros 75 dias por motivos conhecidos (mastite graves ou dados de produção incompletos). Eliminaram-se também lactações com duração inferior a 90 dias. Foram, então, eliminadas 44 lactações, sendo 29 do rebanho "Cova da Onça" e 15 do rebanho "Epamig". Após essa etapa de consistência, foram gerados cinco arquivos para análise, conforme a classe de idade ao parto (CIP), mostrada na Tabela 1.

Com base nos dados diários reais dos primeiros 75 dias de lactação de cada arquivo, calculou-se a produção acumulada real dia-a-dia para esse período.

A produção acumulada estimada com o uso dos fatores foi obtida conforme método oficial (BRASIL, 1986) definido com base no trabalho de Everett \& Carter (1968). Utilizou-se parte da fórmula do cálculo da quantidade total de leite produzida em uma lactação,

Tabela 1 - Freqüência de lactações por classe de idade ao parto (CIP)

Table 1 - Lactations frequency by calving age class (CAC)

\begin{tabular}{|c|c|}
\hline $\begin{array}{l}\text { Classe de idade ao parto } \\
\text { Calving age class }\end{array}$ & $\begin{array}{l}\text { Número de lactações } \\
\text { Number of lactations }\end{array}$ \\
\hline CIP 1 (até 3 anos) & 104 \\
\hline CAC $1(<3$ years $)$ & \\
\hline CIP 2 (de 3,1 a 4 anos) & 56 \\
\hline $\begin{array}{l}\text { CAC } 2 \text { (3.1 to } 4 \text { years) } \\
\text { CIP } 3 \text { (de } 4,1 \text { a } 5 \text { anos) }\end{array}$ & 44 \\
\hline $\begin{array}{l}\text { CAC } 3 \text { ( } 4.1 \text { to } 5 \text { years }) \\
\text { CIP } 4 \text { (de } 5,1 \text { a } 6 \text { anos })\end{array}$ & 18 \\
\hline $\begin{array}{l}\text { CAC } 4 \text { ( } 5.1 \text { to } 6 \text { years) } \\
\text { CIP } 5 \text { (maior que } 6,1 \text { anos) } \\
\text { CAC } 5 \text { (> } 6.1 \text { years) }\end{array}$ & 40 \\
\hline
\end{tabular}


em que a quantidade de leite produzida ao primeiro controle é obtida pelas fórmulas:

$$
\begin{gathered}
\operatorname{PLT}^{\circ} \mathrm{c}=\mathrm{C}_{1} \times \mathrm{E}_{1} \times \mathrm{F}_{1} \\
\mathrm{~F}_{1}=\mu+\mathrm{b}_{1} \cdot \mathrm{E}_{1}^{\mathrm{a}}+\mathrm{b}_{2} \cdot \mathrm{E}_{1}+\mathrm{b}_{3}\left(1 / \mathrm{E}_{1}^{2}\right)
\end{gathered}
$$

em que: $\mathrm{PLT} 1{ }^{\circ} \mathrm{c}$ é a produção total de leite acumulada até o primeiro controle (em $\mathrm{kg}$ ); $\mathrm{C}_{1}$ é a quantidade de leite $(\mathrm{kg})$ obtida no primeiro controle da lactação; $\mathrm{E}_{1}$ é o intervalo em dias, entre a data do parto e a data do primeiro controle da lactação, podendo variar de 6 a 75 dias; $F_{1}$ é o fator de correção para o primeiro controle da lactação; $\mu, b_{1}, b_{2}, b_{3}$ e a são parâmetros do modelo, conforme a idade ao parto (Tabela 2 ).

Como cada CIP utiliza diferentes fatores de correção para o primeiro controle, foram utilizados, em cada arquivo de dados, os respectivos fatores (F1) para o cálculo da produção acumulada, conforme a CIP. Calculou-se, assim, a produção acumulada até o 6o dia após o parto, até o 70 dia e, assim, sucessivamente, até uma produção acumulada ao 75으 dia de lactação para todas as vacas em cada arquivo.

Para avaliar os fatores de Everett \& Carter (1968), criou-se uma estrutura de análise pelo PROC MIXED (Littell et al.,1996), analisando-se, como medidas repetidas, as produções acumuladas de cada lactação ao longo dos dias. Foram utilizados nessa análise os dias de controle 6, 20, 34, 48, 62 e 75 e comparados os valores de produção de leite reais acumulados e os valores acumulados, utilizando-se os fatores de correção para todas as vacas em cada uma das classes de idade ao parto.

A estrutura de covariância utilizada foi aquela com maior valor para o critério de informação de Akaike, considerando as estruturas CS (compound symmetry), UN (unstructured) e AR(1) (first-order autoregressive). O modelo utilizado para a produção de leite acumulada foi:

$$
Y_{i j k}=\mu+V_{i}+M_{j}+V_{i j}+D_{k}+M_{j k}+e_{i j k}
$$

em que: $Y_{i j k}$ é a produção de leite acumulada da vaca i pelo método $\mathrm{j}$, até o dia do controle $\mathrm{k} ; \mu$ é a média geral; $\mathrm{V}_{\mathrm{i}}$ é o efeito da vaca $\mathrm{i}(\mathrm{i}=1,2,3, \ldots, \mathrm{n}) 1$; $\mathrm{M}_{\mathrm{j}}$ é o efeito do método de cálculo da produção acumulada $\mathrm{j}$ ( $\mathrm{j}=1$ e 2); $\mathrm{VM}_{\mathrm{ij}}$ é o efeito da interação da vaca i com o método $j$, sendo usado como erro para testar o efeito do método; $\mathrm{D}_{\mathrm{k}}$ é o efeito do dia do primeiro controle $\mathrm{k}(\mathrm{k}=6,20,34,48,62$ e 75); $\mathrm{MD}_{\mathrm{jk}}$ é o efeito da interação do método $\mathrm{j}$ com o dia do primeiro controle $\mathrm{k}$; $\mathrm{e}_{\mathrm{ijk}}$ é o erro associado a cada observação.

Para o cálculo dos novos fatores, para cada classe de idade ao parto, utilizou-se a mesma metodologia de Everett \& Carter (1968), sendo as produções acumuladas calculadas conforme a equação abaixo:

$$
\sum_{i=1}^{j} \mathrm{Y}_{\mathrm{i}} / \mathrm{JY}_{\mathrm{j}}
$$

em que: $\mathrm{Y}_{\mathrm{i}}$ é a média da produção de leite até o iéssimo dia da lactação; J é o número de dias do período a ser estimado $(j=6, \ldots, 75) ; Y_{j}$ é a média da produção de leite no jéssimo dia da lactação.

Cada fator é a proporção entre o somatório das produções médias até o dia j em relação à produção estimada pela multiplicação da produção num dado dia j por J.

Considerando-se esses fatores, estimaram-se os parâmetros por meio do método modificado de Gauss-

Tabela 2 - Valores dos parâmetros (Everett \& Carter, 1968), em função da idade ao parto, para o cálculo de $F_{1}$ Table 2 - Parameter values (Everett \& Carter, 1968), according to calving class age, for $F_{1}$ estimate

\begin{tabular}{llcccc}
\hline Parâmetro & $\leq 3$ anos & 3,1 a 4 anos & 4,1 a 5 anos & 5,1 a 6 anos & $>6,1$ anos \\
Parameter & $\leq 3$ years & 3.1 to 4 years & 4.1 to 5 years & 5.1 to 6 years & $>6.1$ years \\
\hline $\mathrm{m}$ & 0,8553 & 0,8995 & 0,9103 & 0,9018 & 0,8950 \\
$\mathrm{~b}_{1}$ & 0,0 & 0,002041 & 0,009057 & 0,002561 & 0,002793 \\
$\mathrm{~b}_{2}$ & 0,001933 & $-0,002478$ & $-0,012320$ & $-0,004018$ & $-0,004305$ \\
$\mathrm{~b}_{3}$ & 0,1619 & 0,1090 & 0,1080 & 0,1204 & 0,1192 \\
$\mathrm{a}$ & 0,0 & 1,19 & 1,10 & 1,19 & 1,19 \\
$\mathrm{R}^{2}$ & 0,983 & 0,997 & 0,991 & 0,988 & 0,994 \\
\hline
\end{tabular}


Newton, disponível no procedimento NLIN SAS (1995). Foram utilizadas 70 variáveis por classe de idade de parto ( 60 ao $75^{\circ}$ dia de produção de leite). Os parâmetros iniciais do modelo foram obtidos de Everett \& Carter (1968) ou próximos a esses, utilizando-se a equação:

$$
F_{n}=\mu+b_{1} \cdot E_{1}^{a}+b_{2} \cdot E_{1}+b_{3}\left(1 / E_{1}^{2}\right)
$$

em que: $F_{n}$ é o novo fator gerado; $E_{1}$ é o dia do primeiro controle $(6, \ldots, 75) ; \mathrm{m}, \mathrm{b}_{1}, \mathrm{~b}_{2}, \mathrm{~b}_{3}$ e a são parâmetros do novo modelo.

\section{Resultados e Discussão}

As vacas da classe de idade ao parto até 3 anos (CIP1) apresentaram produção média inicial de 7,2 $(0,3) \mathrm{kg} / \mathrm{dia}$ e as vacas das demais classes apresentaram produções iniciais médias entre 9,3 e 11,4 kg de leite (Tabela 3).

Quanto à produção máxima ou produção no dia do pico, as vacas da CIP 1 apresentaram valores acima dos observados por Grossi (1999), em seu estudo com vacas de vários grupos genéticos Holandês-Zebu, e também superiores aos valores encontrados por Junqueira et al. (1997) em vacas Holandesas, com idade ao parto inferiores a 37 meses. Entretanto, estão bastante próximos aos 27,8 kg/dia estimados pela função gama incompleta obtidos por Lopes (1994) em animais da raça Holandesa de primeira ordem de lactação.
A classe de idade ao parto entre 4 e 5 anos (CIP3) apresentou a maior produção de leite no pico e a maior produção total nos primeiros 75 dias de lactação dentre as estudadas. Segundo Freitas et al. (2000), ocorre um efeito quadrático da idade da vaca ao parto sobre a produção total de leite na lactação, atingindo o máximo aos 78 meses (6,5 anos). Pires (1984) observou efeito semelhante, constatando produção máxima entre 72 e 90 meses de idade ao parto.

O dia do pico ocorreu entre 41,3 e 44,6 dias pós parto (Tabela 3), apresentando valores próximos ao limite mínimo observado por Junqueira et al. (1997), os quais, com base em 2.036 lactações completas no período entre 1989 e 1991, provenientes de registros da Associação de Criadores de Gado Holandês do Estado de Minas Gerais, em 122 rebanhos agrupados em cinco núcleos, concluíram que os picos de produção ocorreram entre 1,5 a 2,1 meses de lactação.

Os valores observados nesse período inicial (Figura 1) são bastante semelhantes aos encontrados na literatura, muito embora, não tenham sido comparados com dados diários de produção, o que demonstra que as lactações utilizadas têm comportamento lactacional próximo aos rebanhos de gado Holandês estudados no Brasil.

Para todas as cinco classes estudadas de idade ao parto, a produção acumulada real até o primeiro controle foi menor $(\mathrm{P}<0,05)$ que a produção estimada pelos fatores de Everett \& Carter (1968). Não foi

Tabela 3 - Comportamento lactacional nos primeiros 75 dias de produção de leite, médias (erro padrão) nas cinco classes de idade ao parto, CIP 1 (= 3 anos), CIP 2 (3,1-4 anos), CIP 3 (4,1-5 anos), CIP 4 (5,1-6 anos) e CIP 5 (> 6,1 anos)

Table 3 - Lactation behavior in the first 75 days of milk production, means and standard error in the five calving age classes, CAC 1 (= 3 years), CAC 2 (3.1-4 years), CAC 3 (4,1-5 years), CAC 4 (5,1-6 years) and CAC 5 (> 6,1 years)

\begin{tabular}{lccccc}
\hline $\begin{array}{l}\text { Classe de idade } \\
\text { ao parto }\end{array}$ & $\begin{array}{c}\text { Número } \\
\text { de lactações } \\
\text { Number of } \\
\text { classes }\end{array}$ & $\begin{array}{c}\text { Produção } \\
\text { no 10 dia } \\
\text { First day } \\
\text { production }\end{array}$ & $\begin{array}{c}\text { Produção no } \\
\text { dia do pico } \\
\text { Production in } \\
\text { the peak day }\end{array}$ & $\begin{array}{c}\text { Dia } \\
\text { do pico } \\
\text { Peak } \\
\text { day }\end{array}$ & $\begin{array}{c}\text { Produção total - } \\
\text { primeiros } 75 \text { dias } \\
\text { Total production- } \\
\text { first } 75 \text { days }\end{array}$ \\
\hline $\begin{array}{l}\text { CIP1 } \\
\text { CAC } 1\end{array}$ & 104 & $7,2(0,3)$ & $26,2(0,5)$ & $43,1(1,7)$ & $1666,9(32,6)$ \\
CIP2 & 56 & $9,3(0,5)$ & $31,8(0,9)$ & $43,3(1,9)$ & $2039,7(56,7)$ \\
CAC 2 & 44 & $10,7(0,6)$ & $35,2(0,9)$ & $44,2(2,1)$ & $2253,2(63,3)$ \\
CIP3 & 18 & $10,6(1,0)$ & $34,1(1,8)$ & $44,6(3,5)$ & $2171,4(121,6)$ \\
CAC 3 & & $11,4(0,6)$ & $32,4(1,1)$ & $41,3(2,3)$ & $2088,6(73,7)$ \\
CIP4 & & & & \\
CAC 4 & 40 & & & &
\end{tabular}

\section{R. Bras. Zootec., v.31, n.2, p.924-933, 2002 (suplemento)}




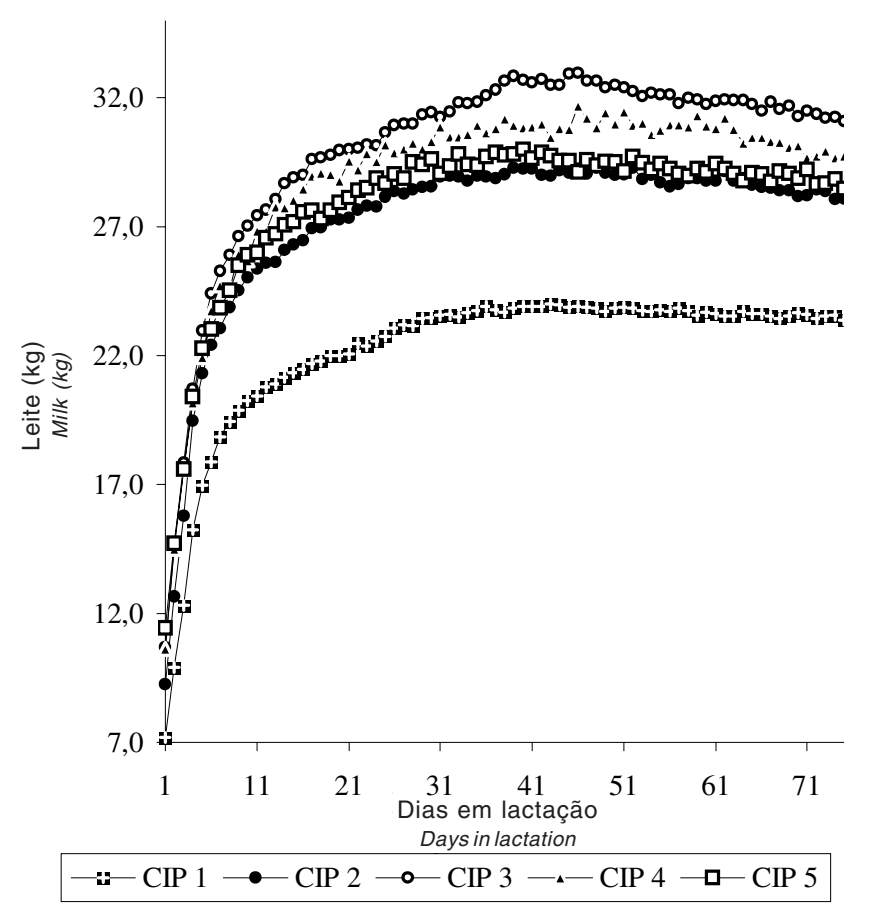

Figura 1 - Comportamento lactacional médio (kg de leite) nos primeiros 75 dias de lactação nas diferentes classes de idade ao parto. CIP 1 ( $\leq 3$ anos), CIP 2 (3,1-4 anos), CIP $3(4,1-5$ anos), CIP 4 (5,1-6 anos) e CIP 5 (> 6,1 anos).

Figure 1 - Lactation behavior (milk $\mathrm{kg}$ ) in the first 75 days of milk production, in the different calving age classes, CAC 1 ( $\leq 3$ years), CAC 2 (3.1-4 years), CAC 3 (4.1-5 years), CAC 4 (5.1-6 years) and $C A C 5$ (> 6.1 years).

encontrada interação $(\mathrm{P}>0,05)$ entre método de cálculo da produção acumulada $\mathrm{x}$ dia do primeiro controle, exceto para a CIP 2.

Por esses resultados infere-se que, nos rebanhos estudados, os fatores de ajuste para o primeiro controle utilizados atualmente superestimaram, em todas as classes de idade ao parto, a produção de leite acumulada. $\mathrm{O}$ erro relativo apresenta maiores valores no início da lactação (primeiro controle no 60 dia). Mas, como a produção acumulada é referente a apenas 6 dias, o erro é quantitativamente, pequeno (Tabela 4).

Com o avanço do dia do primeiro controle ( 6 , , $20^{\mathrm{o}}, 34^{\mathrm{o}}, 48^{\mathrm{o}}, 62^{\circ} \mathrm{o}$ e $75^{\mathrm{o}}$ dia de produção de leite), ocorre uma queda no erro relativo de superestimação. Entretanto, o impacto do dia do

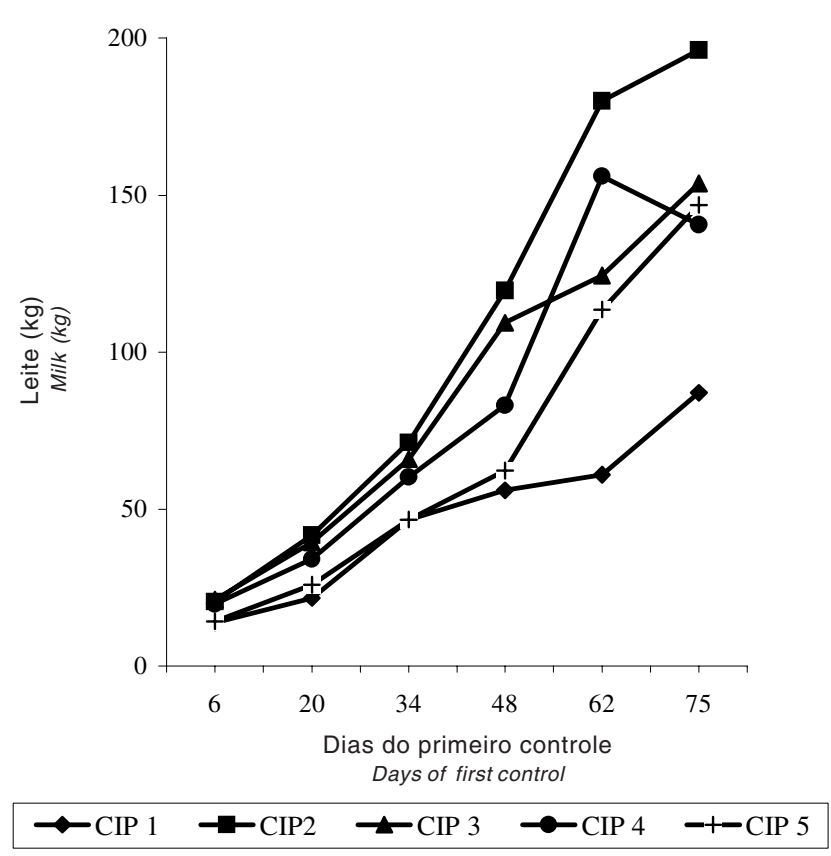

Figura 2 - Diferença entre a produção acumulada estimada pelos fatores oficiais e a produção acumulada real em diferentes dias de primeiro controle, em $\mathrm{kg}$ de leite, nas diferentes classes de idade ao parto (CIP)

Figure 2 - Difference between estimated accumulate production by official factors and real accumulate production in different days of first control $(\mathrm{kg}$ of milk), in the different calving age classes (CAC)

primeiro controle mais tardio na quantidade de leite superestimada é maior (Figura 2).

A classe de idade ao parto 1 (até 3 anos) apresenta uma superestimativa entre 13,4 e $87,1 \mathrm{~kg}$ de leite, quando o primeiro controle foi ao $6^{\circ}$ ou o $75^{\circ}$ dia, respectivamente. Na classe de idade ao parto 2, esses valores variam de 20,6 e 196,3 $\mathrm{kg}$ de leite nos mesmos dias de primeiro controle.

As classes 3, 4 e 5 apresentaram valores intermediários aos das classes 1 e 2 e, em geral, seguem o mesmo comportamento dessas.

Como não foram feitas comparações estatísticas entre classes de idade ao parto pelo fato de utilizarem fatores de correção diferentes, não é possível concluir se as quantidades apresentadas indicam uma superestimação diferenciada entre classe de idade ao parto. 
Tabela 4 - Médias ajustadas das produções de leite acumuladas reais e estimadas pelo fator e superestimação dos fatores de correção em relação à produção real, em kg de leite e em termos percentuais, de acordo com os diferentes dias do primeiro controle, para as cinco classes de idade ao parto (CIP)

Table 4 - Adjusted means of the accumulated milk production (real and estimated by factor) and overestimating of the correction factors in relation to real production (milk $\mathrm{kg}$ and \%), according to different days of first control, for the five calving age classes (CAC)

\begin{tabular}{|c|c|c|c|c|c|c|}
\hline \multirow{2}{*}{$\begin{array}{l}\text { Classes de idade } \\
\text { ao parto } \\
\text { Calving age } \\
\text { classes }\end{array}$} & \multirow{2}{*}{$\begin{array}{l}\text { Número de } \\
\text { lactações } \\
\text { Number } \\
\text { oflactations }\end{array}$} & \multirow{2}{*}{$\begin{array}{c}\text { Dia do primeiro } \\
\text { controle } \\
\text { First day } \\
\text { production }\end{array}$} & \multirow{2}{*}{$\begin{array}{l}\text { Produção real } \\
\text { acumulada }(\mathrm{kg}) \\
\text { Accumulated real } \\
\text { production }(\mathrm{kg})\end{array}$} & \multirow{2}{*}{$\begin{array}{l}\text { Produção estimada } \\
\text { acumulada (kg) } \\
\text { Accumulated estimated } \\
\text { production }(\mathrm{kg})\end{array}$} & \multicolumn{2}{|c|}{$\begin{array}{l}\text { Diferença } \\
\text { Difference }\end{array}$} \\
\hline & & & & & $\begin{array}{c}\text { Leite }(\mathrm{kg}) \\
\text { Milk }(\mathrm{kg})\end{array}$ & $\%$ \\
\hline CIP1 & 104 & 6 & 79,5 & 93,4 & 13,9 & 17,5 \\
\hline$\leq 3$ anos & & 20 & 371,4 & 393,2 & 21,8 & 5,9 \\
\hline$C A C 1$ & & 34 & 694,3 & 740,9 & 46,7 & 6,7 \\
\hline \multirow[t]{3}{*}{$\leq 3$ years } & & 48 & 1028,5 & 1084,5 & 56,0 & 5,4 \\
\hline & & 62 & 1360,7 & 1421,7 & 60,9 & 4,5 \\
\hline & & 75 & 1666,9 & 1753,9 & 87,1 & 5,2 \\
\hline CIP2 & 56 & 6 & 100,9 & 121,5 & 20,6 & 20,4 \\
\hline $3,1-4$ anos & & 20 & 461,3 & 503,1 & 41,8 & 9,1 \\
\hline$C A C 2$ & & 34 & 858,0 & 929,1 & 71,2 & 8,3 \\
\hline \multirow[t]{3}{*}{$3.1-4$ years } & & 48 & 1265,4 & 1385,1 & 119,7 & 9,5 \\
\hline & & 62 & 1670,2 & 1850,1 & 180,0 & 10,8 \\
\hline & & 75 & 2039,7 & 2235,9 & 196,3 & 9,6 \\
\hline CIP 3 & 44 & 6 & 111,2 & 132,4 & 21,2 & 19,1 \\
\hline $4,1-5$ anos & & 20 & 504,9 & 544,3 & 39,4 & 7,8 \\
\hline CAC3 & & 34 & 938,0 & 1003,9 & 66,0 & 7,0 \\
\hline \multirow[t]{3}{*}{$4.1-5$ years } & & 48 & 1393,9 & 1503,3 & 109,3 & 7,8 \\
\hline & & 62 & 1843,3 & 1967,8 & 124,5 & 6,8 \\
\hline & & 75 & 2253,2 & 2407,0 & 153,8 & 6,8 \\
\hline CIP 4 & 18 & 6 & 108,6 & 128,5 & 19,9 & 18,3 \\
\hline $5,1-6$ anos & & 20 & 491,2 & 525,2 & 34,0 & 6,9 \\
\hline CAC4 & & 34 & 912,5 & 972,8 & 60,3 & 6,6 \\
\hline \multirow{3}{*}{$5.1-6$ years } & & 48 & 1345,6 & 1428,7 & 83,1 & 6,2 \\
\hline & & 62 & 1779,8 & 1935,8 & 155,9 & 8,8 \\
\hline & & 75 & 2171,4 & 2312,0 & 140,6 & 6,5 \\
\hline CIP 5 & 40 & 6 & 109,4 & 123,7 & 14,3 & 13,1 \\
\hline$>6,1$ anos & & 20 & 480,8 & 506,7 & 25,9 & 5,4 \\
\hline$C A C 5$ & & 34 & 887,4 & 934,1 & 46,7 & 5,3 \\
\hline \multirow[t]{3}{*}{$>6.1$ years } & & 48 & 1302,6 & 1364,8 & 62,2 & 4,8 \\
\hline & & 62 & 1712,9 & 1826,5 & 113,6 & 6,6 \\
\hline & & 75 & 2088,6 & 2235,4 & 146,9 & 7,0 \\
\hline
\end{tabular}

Tabela 5 - Valores dos novos parâmetros para os dois rebanhos estudados, em função da idade ao parto, para o cálculo de novos fatores de ajuste para o primeiro controle da lactação $\left(F_{n}\right)$ e o $R^{2}$ dos modelos

Table 5 - Values of the new parameters for studied herds, according to calving age, for estimate the new adjusted factors for the first lactation day $\left(F_{n}\right)$ and $R^{2}$ values of models

\begin{tabular}{lccccc}
\hline Parâmetros & $\leq 3$ anos & 3,1 a 4 anos & 4,1 a 5 anos & 5,1 a 6 anos & $>6,1$ anos \\
Parameters & $\leq 3$ years & $3.1-4$ years & $4.1-5$ years & $5.1-6$ years & $>$ 6.1 years \\
\hline$\mu$ & 0,796737 & 0,804859 & 0,847717 & 0,816170 & 0,802075 \\
$\mathrm{~b}_{1}$ & $-0,000292$ & 0,000035 & 0,023277 & 0,004639 & $-0,000247$ \\
$\mathrm{~b}_{2}$ & 0,002695 & 0,001947 & $-0,026664$ & $-0,004278$ & 0,003287 \\
$\mathrm{~b}_{3}$ & $-3,007927$ & $-2,655315$ & $-3,273936$ & $-2,298975$ & $-1,199275$ \\
$\mathrm{a}$ & 1,175274 & 1,420616 & 1,044836 & 1,072227 & 1,324465 \\
$\mathrm{R}^{2}$ & 0,9885 & 0,9911 & 0,9801 & 0,9772 & 0,9797 \\
\hline
\end{tabular}


Tabela 6 - Fatores de correção estimados para o primeiro controle da lactação de vacas da raça Holandesa para os rebanhos estudados, nas diferentes classes de idade ao parto - CIP

Table 6 - Estimated correction factors for first lactation control in Holstein cows of the studied herds at different calving age class - CAC

\begin{tabular}{|c|c|c|c|c|c|}
\hline $\begin{array}{c}\text { Dias em } \\
\text { lactação } \\
\text { Days in } \\
\text { lactation }\end{array}$ & $\begin{aligned} & \text { CIP } 1 \\
\leq & 3 \text { anos } \\
& \text { CAC1 } \\
\leq & 3 \text { years }\end{aligned}$ & $\begin{array}{c}\text { CIP2 } \\
3,1-4 \text { anos } \\
\text { CAC2 } \\
3.1-4 \text { years }\end{array}$ & $\begin{array}{c}\text { CIP3 } \\
4,1-5 \text { anos } \\
\text { CAC3 } \\
4.1-5 \text { years }\end{array}$ & $\begin{array}{c}\text { CIP4 } \\
5,1-6 \text { anos } \\
\text { CAC5 } \\
5.1-6 \text { years }\end{array}$ & $\begin{aligned} & \text { CIP5 } \\
> & 6,1 \text { anos } \\
& \text { CAC6 } \\
> & 6.1 \text { years }\end{aligned}$ \\
\hline 6 & 0,726 & 0,743 & 0,748 & 0,758 & 0,786 \\
\hline 7 & 0,751 & 0,765 & 0,772 & 0,777 & 0,797 \\
\hline 8 & 0,768 & 0,780 & 0,788 & 0,789 & 0,806 \\
\hline 9 & 0,780 & 0,790 & 0,799 & 0,798 & 0,812 \\
\hline 10 & 0,790 & 0,799 & 0,806 & 0,805 & 0,818 \\
\hline 11 & 0,797 & 0,805 & 0,813 & 0,811 & 0,822 \\
\hline 12 & 0,803 & 0,811 & 0,817 & 0,815 & 0,826 \\
\hline 13 & 0,809 & 0,816 & 0,821 & 0,820 & 0,830 \\
\hline 14 & 0,813 & 0,820 & 0,825 & 0,823 & 0,834 \\
\hline 15 & 0,817 & 0,824 & 0,827 & 0,826 & 0,837 \\
\hline 16 & 0,821 & 0,827 & 0,830 & 0,829 & 0,840 \\
\hline 17 & 0,824 & 0,831 & 0,832 & 0,832 & 0,843 \\
\hline 18 & 0,828 & 0,834 & 0,835 & 0,835 & 0,846 \\
\hline 19 & 0,831 & 0,837 & 0,837 & 0,838 & 0,849 \\
\hline 20 & 0,834 & 0,840 & 0,839 & 0,840 & 0,852 \\
\hline 21 & 0,836 & 0,842 & 0,841 & 0,842 & 0,855 \\
\hline 22 & 0,839 & 0,845 & 0,843 & 0,845 & 0,857 \\
\hline 23 & 0,841 & 0,848 & 0,844 & 0,847 & 0,860 \\
\hline 24 & 0,844 & 0,850 & 0,846 & 0,850 & 0,862 \\
\hline 25 & 0,846 & 0,853 & 0,848 & 0,852 & 0,865 \\
\hline 26 & 0,849 & 0,855 & 0,850 & 0,854 & 0,867 \\
\hline 27 & 0,851 & 0,858 & 0,852 & 0,856 & 0,870 \\
\hline 28 & 0,854 & 0,860 & 0,854 & 0,859 & 0,872 \\
\hline 29 & 0,856 & 0,862 & 0,856 & 0,861 & 0,875 \\
\hline 30 & 0,858 & 0,865 & 0,858 & 0,863 & 0,877 \\
\hline 31 & 0,860 & 0,867 & 0,859 & 0,865 & 0,879 \\
\hline 32 & 0,863 & 0,870 & 0,861 & 0,868 & 0,882 \\
\hline 33 & 0,865 & 0,872 & 0,863 & 0,870 & 0,884 \\
\hline 34 & 0,867 & 0,874 & 0,865 & 0,872 & 0,886 \\
\hline 35 & 0,869 & 0,876 & 0,867 & 0,875 & 0,889 \\
\hline 36 & 0,871 & 0,879 & 0,869 & 0,877 & 0,891 \\
\hline 37 & 0,874 & 0,881 & 0,871 & 0,879 & 0,893 \\
\hline 38 & 0,876 & 0,883 & 0,874 & 0,881 & 0,896 \\
\hline 39 & 0,878 & 0,886 & 0,876 & 0,884 & 0,898 \\
\hline 40 & 0,880 & 0,888 & 0,878 & 0,886 & 0,900 \\
\hline 41 & 0,882 & 0,890 & 0,880 & 0,888 & 0,902 \\
\hline 42 & 0,884 & 0,892 & 0,882 & 0,891 & 0,905 \\
\hline 43 & 0,886 & 0,895 & 0,884 & 0,893 & 0,907 \\
\hline 44 & 0,888 & 0,897 & 0,887 & 0,895 & 0,909 \\
\hline 45 & 0,890 & 0,899 & 0,889 & 0,897 & 0,911 \\
\hline 46 & 0,893 & 0,901 & 0,891 & 0,900 & 0,913 \\
\hline 47 & 0,895 & 0,904 & 0,893 & 0,902 & 0,916 \\
\hline 48 & 0,897 & 0,906 & 0,896 & 0,904 & 0,918 \\
\hline 49 & 0,899 & 0,908 & 0,898 & 0,907 & 0,920 \\
\hline 50 & 0,901 & 0,910 & 0,900 & 0,909 & 0,922 \\
\hline 51 & 0,903 & 0,913 & 0,903 & 0,912 & 0,924 \\
\hline 52 & 0,905 & 0,915 & 0,905 & 0,914 & 0,926 \\
\hline 53 & 0,907 & 0,917 & 0,908 & 0,916 & 0,928 \\
\hline 54 & 0,909 & 0,919 & 0,910 & 0,919 & 0,930 \\
\hline 55 & 0,911 & 0,922 & 0,913 & 0,921 & 0,933 \\
\hline 56 & 0,913 & 0,924 & 0,915 & 0,923 & 0,935 \\
\hline 57 & 0,915 & 0,926 & 0,918 & 0,926 & 0,937 \\
\hline 58 & 0,917 & 0,928 & 0,920 & 0,928 & 0,939 \\
\hline 59 & 0,920 & 0,931 & 0,923 & 0,931 & 0,941 \\
\hline 60 & 0,922 & 0,933 & 0,925 & 0,933 & 0,943 \\
\hline
\end{tabular}

R. Bras. Zootec., v.31, n.2, p.924-933, 2002 (suplemento) 
Continuação da Tabela $6 . .$.

\begin{tabular}{cccccc}
\hline Dias em & CIP1 & CIP2 & CIP3 & CIP4 & CIP5 \\
lactação & $\leq 3$ anos & $3,1-4$ anos & $4,1-5$ anos & 5,1-6 anos & CAC5 \\
$\begin{array}{c}\text { Days in } \\
\text { lactation }\end{array}$ & CAC1 & CAC2 & CAC3 & CAC6 \\
\hline 61 & $\leq$ years & $3.1-4$ years & $4.1-5$ years & $5.1-6$ years & $>$ years \\
62 & 0,924 & 0,935 & 0,928 & 0,935 & 0,945 \\
63 & 0,926 & 0,937 & 0,930 & 0,938 & 0,947 \\
64 & 0,928 & 0,940 & 0,933 & 0,940 & 0,949 \\
65 & 0,930 & 0,942 & 0,936 & 0,943 & 0,951 \\
66 & 0,932 & 0,944 & 0,938 & 0,945 & 0,953 \\
67 & 0,934 & 0,946 & 0,941 & 0,950 & 0,955 \\
68 & 0,936 & 0,949 & 0,944 & 0,953 & 0,957 \\
69 & 0,938 & 0,951 & 0,947 & 0,955 & 0,959 \\
70 & 0,940 & 0,953 & 0,949 & 0,958 & 0,961 \\
71 & 0,942 & 0,956 & 0,955 & 0,960 & 0,963 \\
72 & 0,944 & 0,958 & 0,958 & 0,963 & 0,965 \\
73 & 0,946 & 0,960 & 0,960 & 0,965 & 0,967 \\
74 & 0,948 & 0,962 & 0,963 & 0,968 & 0,969 \\
75 & 0,950 & 0,965 & 0,966 & 0,970 & 0,971 \\
\hline
\end{tabular}

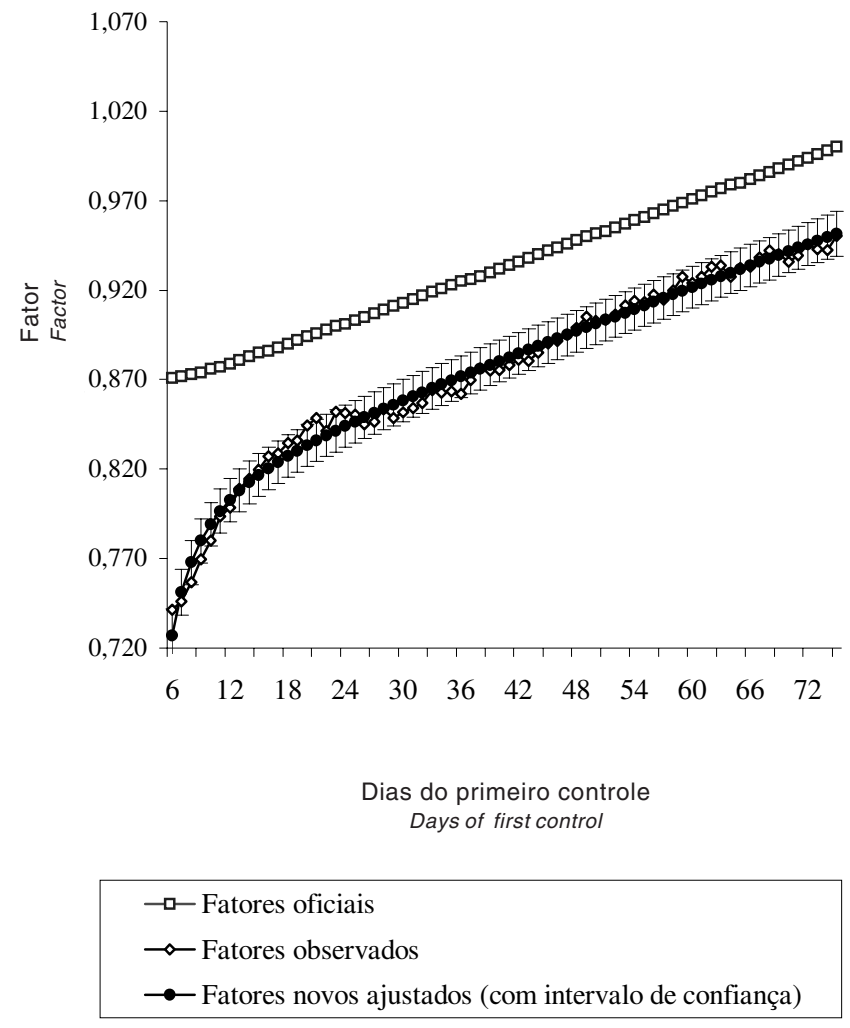

Figura 3 - Fatores de ajuste para o primeiro controle da lactação, classe de idade ao parto 1 (até 3 anos), com base em 104 lactações.

Figure 3 - Adjusted factors to the first lactation control, calving age class (at 3 years), based on 104 lactations.
Os parâmetros das equações para estimar os novos fatores de correção para o primeiro controle (Fn) encontram-se na Tabela 5. Por meio desses parâmetros, podem-se calcular os fatores de ajuste para o primeiro controle (apresentados na Tabela 6), segundo a equação:

$$
F_{n}=\mu+b_{1} \cdot D_{1}^{a}+b_{2} \cdot D_{1}+b_{3}\left(1 / D_{1}^{2}\right)
$$

em que: $\mathrm{F}_{\mathrm{n}}$ é o novo fator de correção para o primeiro controle da lactação; $\mathrm{D}_{1}$ é o intervalo em dias, entre a data do parto e a data do primeiro controle da lactação, podendo variar de 6 a 75 dias; $\mu, b_{1}, b_{2}, b_{3}$ e a são os parâmetros do modelo, conforme idade ao parto.

As equações geradas pelos novos parâmetros apresentam ajustes elevados e o $\mathrm{R}^{2}$ dos modelos foram superiores a 0,97 para as cinco classes estudadas (Tabela 5). Por meio desses valores infere-se que o modelo é capaz de gerar bons fatores de ajuste para o primeiro período de lactação. Na verdade, o modelo, ou seja, a equação proposta por Everett \& Carter (1968) é confiável, desde que os dados utilizados para estimar os parâmetros sejam compatíveis aos rebanhos onde se deseja estimar a produção. No caso do Brasil, essa avaliação não foi feita e os parâmetros do modelo foram simplesmente implementados sem uma avaliação do nível de produção e do comportamento no início da lactação do rebanho nacional.

Observa-se pela Figura 3 que os novos fatores gerados são inferiores aos fatores oficiais em todas 
as classes de idade. Diante desse fato, pode-se afirmar que os fatores atuais estão superestimando a produção de leite até o primeiro controle. Uma vez que os fatores reais estão abaixo dos oficiais, quanto maior for a produção da vaca no dia do primeiro controle, maior a superestimativa e quanto maior o número de dias, maior a superestimação, principalmente se o controle ocorrer próximo ao pico de lactação.

As diferenças numéricas entre os fatores nas diferentes CIPs seguem uma tendência bastante uniforme nas diferentes classes de idade. As maiores diferenças entre os fatores atuais e os novos ocorrem até o 20 ㅇ dia da lactação, oscilando pouco a partir desse ponto nas cinco classes de idade ao parto.

Embora os fatores gerados neste trabalho não apresentem valores definitivos, uma vez que a amostragem foi restrita a apenas dois rebanhos, os fatores utilizados atualmente também não deveriam ser utilizados para a raça Holandesa ou generalizados para outras raças leiteiras no Brasil, porque os mesmos são originados de apenas um rebanho dos Estados Unidos, com um número relativamente pequeno de lactações e com dados de mais de 30 anos atrás.

Os fatores novos não foram comparados a outros, uma vez que nenhum estudo relativo a fatores de ajuste do primeiro controle com dados de produção diária, na raça Holandesa ou outra, foi desenvolvido no Brasil nos últimos anos.

Considerando-se que os novos fatores tenham sido estimados com base nas produções médias diárias e médias de produção acumulada para cada classe de idade ao parto e que existem variações no nível de produção dentro das classes, os novos fatores permitem boas estimativas para os animais que se aproximam mais da média de produção da sua classe de idade ao parto.

\section{Conclusões}

Os fatores de correção utilizados atualmente na fase inicial da lactação pela ABCBRH superestimam a produção de leite acumulada até o primeiro controle para todas as classes de idade ao parto nos rebanhos estudados.
A produção de leite em até 305 dias (P305) é afetada pelo dia do primeiro controle.

Os parâmetros da equação do fator de correção para o primeiro controle, estimados com base em dados de controle diário, apresentaram um bom ajuste do modelo.

Pelo que se observou, fica evidente a necessidade de mais estudos para a estimação desses fatores para as raças leiteiras no Brasil.

\section{Agradecimento}

Aos proprietários e responsáveis técnicos pelas fazendas "Cova da Onça" (Agropecuária Cova da Onça Ltda) e "EPAMIG" (Fazenda Experimental da Empresa de Pesquisa Agropecuária de Minas Gerais), pela concessão dos dados utilizados neste estudo.

\section{Literatura Citada}

BRASIL. Ministério da Agricultura. Secretaria Nacional de Produção Agropecuária. Portaria no 45, de 10 de outubro de 1986. Normas técnicas para execução do serviço de controle leiteiro em bovídeos. Diário Oficial [da] República Federativa do Brasil. Brasília, v.124, n.198, p.15532-15535, out. 1986. Seção 1.

EVERETT, R.W.; CARTER, H.W. Accurracy of test interval method of calculating dairy herd improvement association records. Journal of Dairy Science, v.51, n.12, p.19361941, 1968.

FREITAS, A.F.; DURÃES, M.C.; TEIXEIRA, N.M. Parâmetros genéticos da produção de leite de animais da raça Holandesa mantidos em sistema intensivo do tipo "free stall". Revista Brasileira de Zootecnia, v.9, n.6, p.2008-2012, 2000 (Suplemento 1).

GROSSI, S.F. Eficiência reprodutiva e produtiva em rebanhos leiteiros monitorados por sistema de informação. Jaboticabal: Universidade Estadual Paulista, 1999. 87p. Dissertação (Mestrado em Zootecnia) - Universidade Estadual Paulista, 1999.

JUNQUEIRA, L.V.; NEIVA, R.S.; VEIGA, R.D. et al. Estudo das curvas de lactação de vacas Holandesas de alguns rebanhos do Estado de Minas Gerais, por intermédio de uma função gama incompleta. Revista Brasileira de Zootecnia, v.26, n.6, p.1109-1118, 1997.

LITTELL, R.C.G.A.; MILLIKEN, W.W.; WOLFINGER, R.D. $S A S^{\circledR}$ System for mixed models. Cary, North Caroline: SAS Institute, 1996. 633p.

LOPES, M.A. Estudo da curva de lactação de um rebanho de gado Holandês variedade preto e branco mantido em um sistema intensivo de produção. Lavras: Escola Superior de Agricultura de Lavras, 1994. 42p. Dissertação (Mestrado em Zootecnia) - Escola Superior de Agricultura de Lavras, 1994. 
MACHADO, S.G. Parâmetros genéticos e de ambiente da produção de leite no dia do controle da primeira lactação de vacas da raça Holandesa. Jaboticabal: Universidade Estadual Paulista, 1997. 76p. Dissertação (Mestrado em Zootecnia) - Universidade Estadual Paulista, 1997.

PIRES, A.V. Fatores genéticos e ambiente que afetam a produção de leite e duração da lactação de um rebanho com diferentes grupos genéticos Holandês:Guzerá. Piracicaba: Escola Superior de Agricultura "Luiz de Queiroz”, 1984.112p. Dissertação (Mestrado em Agronomia) - Escola Superior de Agricultura "Luiz de Queiroz", 1984.
SAS INSTITUTE. SAS/STAT. User's guide. Version 6.12 . 4.ed. Cary: 1995. v.2. 1686p.

Recebido em: 29/06/01

Aceito em: 09/01/02 